\title{
Role of the Mastoid in Middle Ear Pressure Regulation
}

\author{
Cuneyt M. Alper, M.D. ${ }^{1}$, Dennis J. Kitsko, D.O. ${ }^{1}$, J. Douglas Swarts, Ph.D. ${ }^{1}$, Brian Martin, \\ D.M.D. ${ }^{2}$, Sancak Yuksel, M.D. ${ }^{3}$, Brendan M. Cullen Doyle ${ }^{1}$, Richard J. M. Villardo, M.D. ${ }^{1}$, \\ and William J. Doyle, Ph.D. ${ }^{1}$ \\ ${ }^{1}$ Division of Pediatric Otolaryngology, Children's Hospital of Pittsburgh of UPMC, Department of \\ Otolaryngology, University of Pittsburgh School of Medicine \\ ${ }^{2}$ Division of Pediatric Dentistry, Children's Hospital of Pittsburgh of UPMC, University of \\ Pittsburgh School of Dental Medicine \\ ${ }^{3}$ Division of Pediatric Otolaryngology, Department of Otorhinolaryngology-Head \& Neck Surgery, \\ University of Texas Medical School at Houston
}

\begin{abstract}
Objective-Determine the role of mastoid volume in middle ear pressure (MEP) regulation. The hypothesis was that inert gas exchange between blood and middle ear (ME) is slower for larger mastoid volumes.

\section{Study Design-Prospective}

Methods-For 21 enrolled subjects, the bilateral surface areas and volumes of the mastoid and tympanum were measured from CT scans in 20 with a wide range of mastoid volumes. Then, 19 subjects were reclined in a chair, fitted with a non-rebreathing mask and breathed room air for 20 minutes (acclimation), a gas composition of $25 \% \mathrm{~N} 2 \mathrm{O}, 20 \% \mathrm{O} 2$, balance $\mathrm{N} 2$ for 30 minutes (experiment) and room air for 30 minutes (recovery). Bilateral MEPs were recorded by tympanometry every 2 minutes. The slopes of the MEP-time functions during N20 breathing were calculated to the first observation of Eustachian tube opening and divided by the estimated bloodME N2O gradient to yield a N2O time-constant. Sufficient data were available for 16 right and 11 left MEs to calculate the time-constant.
\end{abstract}

Results-MEP did not change during the baseline period but, within 10 minutes of breathing the $\mathrm{N} 2 \mathrm{O}$ mixture, showed a progressive increase. The right-left correlation for the time-constant was 0.87 ( $\mathrm{n}=10$ ears, $\mathrm{p}=0.001$ ). Regression of the time-constants on ME volume showed an inverse relationship $(n=23$ ears, $r=-41, p=0.05)$. A better data fit was the curvilinear relationship predicted by a mathematical model of the mastoid acting as a ME ear gas reserve.

Conclusion-These results support the tested hypothesis that the mastoid could serve as ME gas reserve.

\section{Keywords}

adult humans; transmucosal time-constant; $\mathrm{N} 2 \mathrm{O}$ exchange; middle ear; mastoid

Correspondence To: Cuneyt M. Alper, M.D., Children's Hospital of Pittsburgh of UPMC, One Children's Hospital Drive, 4401 Penn Avenue, Pittsburgh, PA 15224, Phone: 412 692-7003, FAX: 412 692-6074, Alperc @pitt.edu.

Presented at: American Society of Pediatric Otolaryngology Meeting, Las Vegas, NV, April 30-May 2, 2010.

Financial Disclosure Information: The authors have no financial disclosures.

Conflict of Interest: The authors have no conflicts to disclose. 


\section{INTRODUCTION}

The efficiency of the middle ear (ME) for coupling tympanic membrane vibrations to mechanical pressures acting on the oval window is inversely related to the absolute value of the ME-ambient pressure deviation. Because the ME is a relatively fixed-volume, temperature-stable, biological gas pocket, its pressure is proportional to the contained gas moles. By extension, the rate and trajectory of ME pressure (MEP) change reflects the summed rates of the loss or gain of the physiological gases $(\mathrm{H} 2 \mathrm{O}, \mathrm{N} 2, \mathrm{O} 2$ and $\mathrm{CO} 2)$ due to exchange with adjacent compartments ${ }^{1}$.

The ME exchanges gases with four compartments, the inner ear via the round window membrane, the ambient environment via the tympanic membrane2, the local blood via the ME mucosa $(\mathrm{MEM})^{3}$ and the nasopharynx via the Eustachian tube (ET)4. Gas exchange across the first three pathways is a passive, partial-pressure gradient-driven diffusive exchange across an inert barrier5, while the fourth pathway is an active, total pressure gradient-driven, bolus exchange of mixed gases in the air-phase4. Because of the physiological ME-local blood partial-pressure gradients6, the net effect of the passive gas transfers is to deplete ME gas volume and progressively lower its pressure (ref. ambient), while that of transET gas transfer is to add sufficient gas volumes to rebalance ME and ambient pressures ${ }^{1}$.

Measurements of the volume gas exchange across the three passive barriers at physiological gradients in animals showed that the transMEM route dominates the exchange kinetics for individual gases and drives the total MEP behavior between ET openings2, 3,5 . Experiments that measured transMEM exchange for the physiological gases in monkeys showed that the exchange of reactive gases $(\mathrm{O} 2$ ad $\mathrm{CO} 2)$ is relatively fast and diffusionlimited, while that of the inert gas, N2, is very slow and perfusion-limited ${ }^{3}$. Because the only substantial physiological gradient to drive transMEM gas exchange is an excess ME N2 pressure relative to blood6, the rate of transMEM N2 exchange is the main determinant of the rate of total MEP decrease between ET openings3. Thus, an accurate estimate of the transMEM N2 exchange rate is fundamental to understanding the demand for gas placed upon the ET and to determining the efficiency of MEP-regulation under different extant conditions.

The ME can be anatomically and functionally subdivided into two communicating airspaces, the anterior tympanum and the posterior mastoid air-cell system (MACS). The tympanum is essentially a large air-cell that contains the ME ossicles and functions as the peripheral transducer organ for hearing. In contrast, the MACS is a multiply partitioned, cellular, airspace that increases ME volume and surface area, but does not participate directly in sound transduction. While the function of the MACS is debated7, numerous studies show that MACS volume is indirectly related to the predisposition of the ME to certain pathological conditions including cholesteatoma and otitis media8, 9. One hypothesis advanced to explain this relationship is that the MACS functions as a ME gas reserve such that MEs with larger MACS require less frequent ET openings to maintain near-ambient total pressure7. This mechanism requires that the rate of transMEM N2 exchange per ME volume is greater for the tympanum than for the MACS. However, geometric considerations as reflected in the surface area volume ratios for the two compartments (MACS>tympanum) show that this mechanism requires that the blood perfusion rate per surface area is less for the MACS when compared to the tympanum ${ }^{10}$.

At physiological partial-pressure gradients, the rate of transMEM N2 exchange is not easily measured due to its extremely slow rate of exchange ${ }^{3}$. To overcome this difficulty, the more tissue-soluble, perfusion-limited, inert gas, N2O, has been used to study transMEM inert gas 
exchange ${ }^{5,11-13}$. Previously, an increase in MEP was reported for anesthetized patients and monkeys breathing gas mixtures that included $\mathrm{N}_{2} \mathrm{O}^{12}, 14$ and that response was used to compare the transMEM inert gas exchange rates between ears with and without pneumatized MACS regions ${ }^{7}, 11$. However, the majority of these data was acquired in the surgical setting with limited control over confounding variables such as gas mixture, blood gas partialpressures, body temperature and frequency of ET openings.

Previously, a protocol for measuring the rate of blood to $\mathrm{ME} \mathrm{N2O} \mathrm{exchange} \mathrm{in} \mathrm{anesthetized}$ monkeys under well-controlled laboratory was described ${ }^{12}$ and a formal mathematical analysis of the underlying physiology was developed that prescribes the requisite conditions for deriving time-constants for transMEM N2O and N2 exchanges from the resulting data ${ }^{13}$. The transMEM time-constant for a gas is defined as the rate of change in ME gas partialpressure divided by its transMEM partial-pressure gradient and is a normalized measure of the partial-pressure change at any specified gradient. This report describes the adaptation of those methods to study transMEM inert gas exchange in non-sedated human subjects, presents estimates for the $\mathrm{N} 2 \mathrm{O}$ and $\mathrm{N} 2$ transMEM time-constants, and tests the hypothesis that the MACS acts a gas reserve in healthy, adult human volunteers.

\section{MATERIALS AND METHODS}

\section{Study Population}

The protocol was approved by the Institutional Review Board at the University of Pittsburgh. Healthy adult subjects were recruited by advertisement and all interested subjects signed an institutionally approved informed consent for study participation. Presenting subjects provided a short medical history; were given a standard Ear Nose and Throat examination; had bilateral tympanometry, and women had a urine pregnancy test. Pregnant women and persons presenting with chronic illnesses; or who were currently taking prescription medications for any condition other than birth control; or with extant unilateral or bilateral otitis media or low tympanic membrane compliance, or with a previous adverse reaction to breathing gas mixtures containing $\mathrm{N} 2 \mathrm{O}$ were excluded. Twenty-one subjects (age range $=20$ to 40 , average age $=26.2 .0 \pm 6.2$ years; 7 Males) were enrolled. Twenty subjects had the CT done but one of these did not complete the breathing experiment. Eighteen subjects completed the breathing experiments, but 2 did not have CT.

\section{Protocols}

Twenty subjects with an expectedly wide range of MACS volumes based on the variability in their MACS surface area measured from a Schuller projection X-ray were selected for study. Each had a CT scan of the ME in the transverse plane at a resolution of $.031 \mathrm{~mm} /$ pixel and a slice thickness of $0.63 \mathrm{~mm}$ using a GE LightSpeed VCT system (General Electric Health Care). No ME pathologies were noted on the CT scans for any subject.

From each CT scan, a set of transverse images through the bilateral MACS regions at 0.25 $\mathrm{cm}$ intervals was selected for study. For the tympanum, every image was included in the reconstruction (i.e. interval=.63 mm). Using Image J software (http://rsbweb.nih.gov/ij/), these sections were imported, and the left and right MACS and tympanums were identified, segmented out and analyzed. For each MACS and tympanum section, the perimeter and area of all air-cells were highlighted, measured, summed across images and multiplied by the section interval to yield MACS and tympanum surface areas $\left(\mathrm{cm}^{2}\right)$ and volumes $(\mathrm{ml})^{10}$. (See Figure 1 for an example of a CT section of the ME in the transverse plane and the corresponding extracted and processed image for the left tympanum and MACS in 1 subject). The average volume of the MACS for these 20 subjects was $5.24 \pm 4.76$ (range $=0.65$ to 21.4 ) $\mathrm{ml}$ and of the tympanum was $0.67 \pm 0.12$ (range $=0.48$ to 0.96$) \mathrm{ml}$. 
Eighteen subjects had $\mathrm{N} 2 \mathrm{O}$ breathing experiments done in the Dental Clinic at the Children's Hospital of Pittsburgh. Subjects were seated comfortably in an exam chair that was reclined to approximately 30 degrees to the horizontal and fitted with the finger probe of a pulse oximeter (Massimo RDS1) and with the upper arm cuff of an automated bloodpressure monitor (Critikon Dynamap 1846SX). A plastic anesthesia mask with a nonrebreathing valve (Breath Tech BT9005- Size 5 Adult) was placed over the nose and mouth. The delivery system supplying the mask was configured such that three gas sources could be placed "on-line" to the gas reservoir (breathing bag); room air, 100\% O2 or a N20 gas mixture $(20 \% \mathrm{O} 2,25 \% \mathrm{~N} 20,75 \% \mathrm{~N} 2) . \mathrm{O} 2$ and the $\mathrm{N} 2 \mathrm{O}$ gas mixture were supplied as tank gas at certified composition (airgas.com) and were pressure regulated to near ambient at the gas reservoir.

The experiment consisted of 3 sequential phases: a 20-minute acclimation period (Period 1, room air breathing); a 30-minute experimental period (Period 2, gas mixture breathing), and a 30-minute recovery period (Period 3, room air breathing). At the completion of the experiment, all subjects breathed $100 \% \mathrm{O} 2$ for a minimum of 5 minutes. Throughout the 80 minutes of the experiment represented by Periods 1-3, bilateral MEP was recorded by tympanometry at 2-minute intervals. For safety, $\mathrm{O} 2$ saturation, heart-rate and blood-pressure were checked at 5-minute intervals. At the end of the $\mathrm{O} 2$ breathing, the subjects were given a brief physical examination and, if recovered from the effects of breathing the $\mathrm{N} 2 \mathrm{O}$ gas mixture, were released from the study.

\section{Statistical Methods}

For Period 2, the MEP data to the first observation of an ET opening were regressed on time to calculate a slope representing the change in MEP per change in time $\left(\delta \mathrm{P}^{\mathrm{ME}} / \delta \mathrm{t}\right)$. This was attributed to the rate of change in ME N2O pressure $\left(\delta \mathrm{P}^{\mathrm{ME}}{ }_{\mathrm{N} 2 \mathrm{O}} / \delta \mathrm{t}\right)$ secondary to gas transfers in response to the established $\mathrm{N} 2 \mathrm{O}$ pressure gradient between blood and $\mathrm{ME}, \mathrm{G}_{\mathrm{N} 2 \mathrm{O}}$. That gradient was estimated as previously described and was assumed to be constant for Period $2^{12}$. Because the rate of change in ME N2O pressure is a linear function of the $\mathrm{N} 2 \mathrm{O}$ pressure gradient, dividing the rate of change in MEP by the estimated gradient $\left[\left(\mathrm{SP}_{\mathrm{N} 2 \mathrm{O}} / \delta \mathrm{t}\right) / \mathrm{G}_{\mathrm{N} 2 \mathrm{O}}\right]$ yields a time-constant with units of pressure/time/pressure. The time-constant is a standardized measure of the rate of ME pressure change. The MACS will serve as a gas reserve if the time-constant decreases with increasing ME (i.e. MACS) volume and as a gas sink if the time constant increases with increasing ME (i.e. MACS) volume. We tested the hypothesis that the MACS serves as a gas reserve by first calculating the slope of the function relating the time-constant to ME volume using least-squares linear regression. Then, we evaluated the significance of this relationship under the hypothesis that the slope was negative and significantly different from a value of 0 (i.e. no relationship) using a 1tailed Student's t test.

The Pearson correlation coefficient was used to evaluate the relatedness of paired variables and the Student's $t$ test was used to evaluate the significance of differences for between group comparisons and for comparisons of the value of a variable with an expected value (e.g. 0). The standard descriptive statistics of average \pm standard deviation and range were used to summarize the data throughout.

\section{RESULTS}

Figure 2a,b shows the MEP-time function for the left and right ears of two subjects, \#18 and \#11 during the gas breathing experiment. Note that for the baseline period (0 to $20 \mathrm{~min}$ ), MEP showed no directional change and varied about a mean value. In contrast, for the experimental period (20-50 minutes) MEP showed an increase interrupted by sharp decreases attributable to ET openings. While the data for subject 18 did not evidence tubal 
openings until late in that period, those for subject 11 were characterized by a repeated series of such events. Period 3 was characterized by a variable response with a trend for all ears to decrease with and without ET openings. There was a sufficiently long period of linear increase to calculate the bilateral slopes of the MEP-time function for subject 18 but this was not true for subject 11 . Of the 20 experiments, a linear period of increase characterized the data for 16 right and 11 left ears (10 bilateral) and the presentation below includes only those ears.

For those 27 ears, the average, standard deviations and range for the slopes of the MEP-time function, the fraction of the total variance in MEP explained by the regression on time $\left(\mathrm{r}^{2}\right)$, and the time-constant were $4.3 \pm 2.2$ (range $=1.2$ to 9.8$) \mathrm{daPa} /$ minute, $0.9 \pm 0.1$ (range $=0.6$ to 1.0 ) and $0.0022 \pm 0.0011$ (range 0.0006 to 0.0050 ) $\mathrm{daPa} / \mathrm{min} / \mathrm{daPa}$, respectively. For the 10 subjects with bilateral data, the time-constants for the left and right ears were significantly correlated $(r=0.87, \mathrm{p}<0.001)$.

Paired data for the time-constant and ME volume were available for 23 ears. Figure 3a shows a scatterplot of the function relating the $\mathrm{N} 2 \mathrm{O}$ time-constant to $\mathrm{ME}$ volume and the solution of the linear regression equation for that function. The data showed a decreasing time-constant with increasing ME volume. The slope of the fitted linear regression equation was significantly different from 0 (slope $=-0.0001$, intercept $=0.0029, r=0.41, p=0.025$ ). However, the general form of the relationship was curvilinear with a decreasing timeconstant that approached an asymptote at larger ME volumes. A curvilinear equation was fitted to the data as previously described and is shown in Figure $3 b^{7}$. The correlation between the observed and predicted time-constants for the linear and curvilinear models was higher for the curvilinear model $(\mathrm{r}=.56)$ than for the linear model $(\mathrm{r}=.41)$ and both were significantly different from a value of 0 ( $\mathrm{p}=0.003$ and 0.025 , respectively).

\section{DISCUSSION}

Increasing MEP during surgical procedures where anesthesia is induced by inhalation of gas mixtures containing high concentrations of $\mathrm{N} 2 \mathrm{O}$ (usually $\approx 50 \%$ ) is a well recognized phenomenon 14,15 . However, data generated in that setting cannot be easily transformed to an estimate of the $\mathrm{N} 2 \mathrm{O}$ time-constant because an implicit assumption underlying the derivation of the time-constant is that the observed change in MEP is wholly attributable to the transfer of $\mathrm{N} 2 \mathrm{O}$ from blood to ME. The validity of this assumption requires that during data collection: 1) the pre-existing, apparent equilibrium between $\mathrm{ME}$ and blood for the physiological gases is not disturbed; 2) $\mathrm{N} 2 \mathrm{O}$ is the only gas with an established ME-blood pressure-gradient (other than those with time-constants too small to affect MEP over short time-intervals; eg. N2);3) the ET does not open to vent gas, and 4) body temperature and MEM blood perfusion remain constant. For a number of reasons including the known effects of anesthesia on body temperature and cardiac output, the unbalanced gas compositions used to induce anesthesia (e.g. high $\mathrm{O} 2$ concentration, multiple non-physiological gases, etc) and the large $\mathrm{N} 2 \mathrm{O}$ concentrations in the breathing mixtures, it is highly unlikely that these assumptions are satisfied in that setting. There, these uncontrolled variables establish multiple transMEM partial-pressure gradients which decouple the rate of MEP change from that for ME N2O.

The principle of using $\mathrm{N} 2 \mathrm{O}$ inhalation to drive a MEP response for purposes of estimating transMEM inert gas time-constants is a viable option in the laboratory setting ${ }^{12}$. There, many of the confounding factors listed above can be avoided or controlled for by the judicious choice of experimental conditions. For example, because there is no requirement for an anesthetic effect, test gas mixtures can be formulated to contain much lower, nonsedating, $\mathrm{N} 2 \mathrm{O}$ concentrations, physiological $\mathrm{O} 2$ concentrations and near physiological $\mathrm{N} 2$ 
concentrations. Breathing this type of gas mixture preserves the pre-existing, apparent blood-ME equilibria for the physiological gases while introducing a well-defined blood-ME gradient for a single test gas, N2O. Repeated assessments of MEP can be scheduled at sufficiently short intervals to capture events typical of ET gas venting and post hoc restrictions can be placed on the use of any data obtained after an ET opening.

Using the rate of change in MEP over the linear pressure increase for Period 2 and the estimated transMEM N2O gradient, a time-constant for transMEM N2O exchange was calculated as the ratio of those two measures. There, $\mathrm{N} 2 \mathrm{O}$ was used as a surrogate inert gas for purposes of estimating the transMEM N2 time-constant. As discussed, the transMEM gas exchange pathway dominates the other passive pathways in effecting MEP change1, and $\mathrm{N} 2$ is the only physiological ME gas not in equilibrium with the local blood6. For these reasons, the transMEM time-constant for N2 defines the time trajectory for ME pressure change and is the single most important determinant of the gas demand placed upon the ET for gas resupply. For a given ME, the transMEM N2 time-constant can be estimated by multiplying the measured transMEM N2O time-constant by the $\mathrm{N} 2: \mathrm{N} 2 \mathrm{O}$ blood solubility ratio ${ }^{5}$. At body temperature $\left(37^{\circ} \mathrm{C}\right)$, the $\mathrm{N} 2: \mathrm{N} 2 \mathrm{O}$ blood solubility ratio is $\approx 0.03$ and thus, from the data presented in the Results section, the average estimated transMEM N2 timeconstant is .00007 with a range of from 0.00002 to $0.00015 \mathrm{daPa} / \mathrm{min} / \mathrm{daPa}$.

The results for the present study show that the $\mathrm{N} 2 \mathrm{O}$ (and N2) time-constant decreases curvilinearly with increasing ME (and MACS) volume to approach an asymptote at a ME volume of approximately $10 \mathrm{ml}$. Because the tympanum volume is relatively fixed while MACS volume was highly variable in this population, this effect can be attributed to the differences in MACS volume. As noted, this pattern is consistent with the hypothesis that the MACS functions as a ME gas reserve. Under those conditions, the frequency of effective ET openings required to prevent pathological under-pressures is decreased and ears with constitutionally moderate or even poor ET function are protected from the development of pathology

A limitation of the study is that only adults were included while otitis media is a disease that primarily affects children in whom the ME geometry and MEP response to breathing gas mixtures has not been studied. This is a goal for future research. Nonetheless, the general protocol and model can be adapted to specific experimental conditions for purposes of testing predictions and/or hypotheses related to MEP-regulation. However, the model requires significant modifications when used to characterize transMEM reactive gas exchange and is not useful for studying transMEM inert or reactive gas exchanges when new exchange compartments (e.g. a ME effusion) are introduced into the simple, 2compartment (blood, ME) model system.

\section{CONCLUSION}

The results show that inert gas time-constants decrease with increasing ME volume which supports the hypothesis that the MACS acts as a gas reserve for the ME. Thus, larger MACS volumes will partially protect the ME from the development of pathological underpressures by allowing for a decreased frequency of ET openings and/or less efficient ET openings when compared to smaller MAC volumes.

\section{Acknowledgments}

Funding: NIH Grant DC007667 
The investigators thank James T. Seroky, M.A. and Julianne Banks, B.S. for assisting with subject recruitment and testing. This study was supported in part by NIH Grant DC007667. William J. Doyle had full access to all the data in the study and takes responsibility for the integrity of the data and the accuracy of the data analysis.

\section{REFERENCES}

1. Kanick SC, Doyle WJ. Barotrauma during air travel: predictions of a mathematical model. J Appl Physiol 2005 May;98(5):1592-1602. [PubMed: 15608090]

2. Yuksel S, Swarts JD, Banks J, Seroky JT, Doyle WJ. In vivo measurement of O(2) and CO(2) gas exchange across the human tympanic membrane. Acta Otolaryngol 2009 Jul;129(7):716-725. [PubMed: 18728916]

3. Doyle WJ, Seroky JT, Alper CM. Gas exchange across the middle ear mucosa in monkeys. Estimation of exchange rate. Arch Otolaryngol Head Neck Surg 1995 Aug;121(8):887-892. [PubMed: 7619416]

4. Cantekin EI, Saez CA, Bluestone CD, Bern SA. Airflow through the eustachian tube. Ann Otol Rhinol Laryngol 1979 Sep-Oct;88(5 Pt 1):603-612. [PubMed: 573980]

5. Ranade A, Lambertsen CJ, Noordergraaf A. Inert gas exchange in the middle ear. Acta Otolaryngol Suppl 1980;371:1-23. [PubMed: 6272533]

6. Hergils L, Magnuson B. Human middle ear gas composition studied by mass spectrometry. Acta Otolaryngol 1990 Jul-Aug;110(1-2):92-99. [PubMed: 2386039]

7. Doyle WJ. The mastoid as a functional rate-limiter of middle ear pressure change. Int J Pediatr Otorhinolaryngol 2007 Mar;71(3):393-402. [PubMed: 17174408]

8. Lesinskas E. Factors affecting the results of nonsurgical treatment of secretory otitis media in adults. Auris Nasus Larynx 2003 Feb;30(1):7-14. [PubMed: 12589843]

9. Valtonen HJ, Dietz A, Qvarnberg YH, Nuutinen J. Development of mastoid air cell system in children treated with ventilation tubes for early-onset otitis media: a prospective radiographic 5-year follow-up study. Laryngoscope 2005 Feb;115(2):268-273. [PubMed: 15689748]

10. Swarts JD, Cullen Doyle BM, Alper CM, Doyle WJ. Surface area-volume relationships for the mastoid air cell system and tympanum in adult humans: implications for mastoid function. Acta Otolaryngol. May; 7.

11. Elam M, Harell M, Luntz M, Fuchs C, Sade J. Middle ear pressure variations during 50\% N2O anesthesia as a function of mastoid pneumatization. Am J Otol 1998 Nov;19(6):709-711. [PubMed: 9831141]

12. Doyle WJ, Banks JM. Middle ear pressure change during controlled breathing with gas mixtures containing nitrous oxide. J Appl Physiol 2003 Jan;94(1):199-204. [PubMed: 12486021]

13. Doyle WJ, Yuksel S, Banks J, Alper CM. Directional asymmetry in the measured nitrous oxide time constant for middle ear transmucosal gas exchange. Ann Otol Rhinol Laryngol 2007 Jan; 116(1):69-75. [PubMed: 17305281]

14. Nader ND, Simpson G, Reedy RL. Middle ear pressure changes after nitrous oxide anesthesia and its effect on postoperative nausea and vomiting. Laryngoscope 2004 May;114(5):883-886. [PubMed: 15126749]

15. Raveh E, Sade J, Mover-Lev H, Guney S. Mastoid buffering properties: I. Gas partial pressures. Ann Otol Rhinol Laryngol 1999 Aug;108(8):750-755. [PubMed: 10453782] 


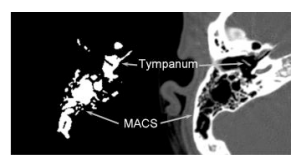

Figure 1.

An example of a transverse CT section through the left ME and the corresponding processed image for the MACS and tympanum in 1 subject. For the processed image, the surface areas and volumes of the MACS and tympanum were calculated by a boundary recognition program. 


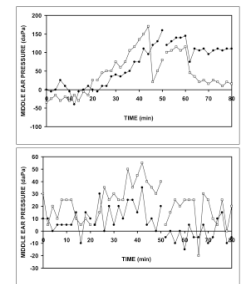

Figure 2.

Left (open squares) and right (closed circles) MEP as a function of time for the N2O exchange experiment on Subject 18 (a) and Subject 11 (b) showing the MEP trajectory for the 3 experimental Periods corresponding to breathing: air (0 to 20 minutes), the N2O mixture (20-50 minutes) and air (50-80 minutes). Note that, for Subject 18 , the monotonic rise in MEP during Period 2 was relatively uninterrupted until approximately 45 and 50 minutes, while for Subject 11, that Period of increase was associated with repeated interruptions. 

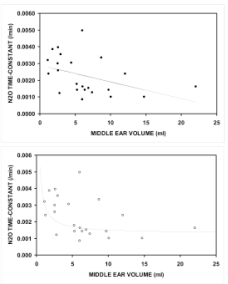

Figure 3.

Scatterplot for the N2O time-constant as a function of ME volume. Solid line is the solution for the linear regression equation (a) and for a fitted curvilinear equation (b). 